\title{
Diffusion Tensor Magnetic Resonance Imaging and Tract- Tracing Analysis of Probst Bundle Structure in Netrin1- and DCC-Deficient Mice
}

\author{
Tianbo Ren, ${ }^{1}$ Jiangyang Zhang, ${ }^{2}$ Celine Plachez, ${ }^{1}$ Susumu Mori, ${ }^{2,3}$ and Linda J. Richards ${ }^{1,4}$ \\ ${ }^{1}$ Department of Anatomy and Neurobiology and The Program in Neuroscience, The University of Maryland School of Medicine, Baltimore, Maryland \\ 21201, 2Department of Radiology, Division of NMR Research, and Department of Biomedical Engineering, Johns Hopkins University School of Medicine, \\ Baltimore, Maryland 21205, ${ }^{3}$ F. M. Kirby Research Center for Functional Brain Imaging, Kennedy Krieger Institute, Baltimore, Maryland 21205, and ${ }^{4}$ The \\ School of Biomedical Sciences and The Queensland Brain Institute, The University of Queensland, Brisbane, Queensland 4072, Australia
}

In many cases of callosal dysgenesis in both human patients and mouse models, misguided fibers from the cortex form abnormal bilateral, barrel-shaped structures known as Probst bundles. Because little is known about how axons are arranged within these anomalous fiber bundles, understanding this arrangement may provide structural and molecular insights into how axons behave when they are misguided in vivo. Previous studies described these bundles as longitudinal swirls of axons that fail to cross the midline (Ozaki et al., 1987). However, recent studies on human acallosal patients using diffusion tensor magnetic resonance imaging (DTMRI) technology suggest that axons project in an anteroposterior direction within the Probst bundle (Lee et al., 2004; Tovar-Moll et al., 2007). This led us to ask the question, is DTMRI an accurate method for analyzing axonal tracts in regions of high axon overlap and disorganization, or is our current perception of axon arrangement within these bundles inaccurate? Using DTMRI, immunohistochemistry, and carbocyanine dye tract-tracing studies, we analyzed the Probst bundles in both Netrin1 and deleted in colorectal cancer (DCC) mutant mice. Our findings indicate that DTMRI can accurately demonstrate fiber tract orientation and morphology where axons are in ordered arrays such as in the dorsal part of the bundle. In ventral areas, where the axons are disorganized, no coordinated diffusion is apparent via DTMRI. In these regions, a higher-resolution approach such as tract tracing is required. We conclude that in DCC and Netrin1 mutant mice, guidance mechanisms remain in the dorsal part of the tract but are lost ventrally.

Key words: corpus callosum; axon guidance; brain development; agenesis of the corpus callosum (ACC); cerebral cortex; magnetic resonance imaging

\section{Introduction}

The corpus callosum is the largest axonal structure in the brain and serves the vital role of coordinating the transfer of information between the two hemispheres. Agenesis of the corpus callosum has been observed in numerous human congenital disorders linked to $>30$ different genes, leading to a variety of physiological, behavioral, and neuropsychiatric deficits (Richards et al., 2004; Paul et al., 2007). The most common structural anomaly associated with human cases of callosal dysgenesis is the presence of large bilateral, barrel-shaped axonal structures. This "longitudinal callosal bundle" was first described by anatomists such as Onufrowicz (1888) and Probst (1901), and today they are commonly called Probst bundles.

\footnotetext{
Received June 19, 2007; revised Aug. 4, 2007; accepted Aug. 7, 2007.

This work was supported by National Health and Medical Research Council (Australia) Grant 456026. L.J.R. was supported by a Senior Research Fellowship from the National Health and Medical Research Council (Australia). We thank Dr. Randal Moldrich for helpful comments on this manuscript.

Correspondence should be addressed to Dr. Linda J. Richards, The University of Queensland School of Biomedical Sciences and The Queensland Brain Institute, Otto Hirschfeld Building, Room 715, Brisbane, Queensland 4072, Australia.E-mail: richards@uq.edu.au.

DOI:10.1523/JNEUROSCI.2787-07.2007

Copyright $\odot 2007$ Society for Neuroscience $\quad 0270-6474 / 07 / 2710345-05 \$ 15.00 / 0$
}

Although Probst bundles are by far the most common pathological finding in human acallosal patients, fiber trajectories within these structures remain poorly understood. As early as 1968, Rakic and Yakovlev (1968) conjectured that cortical axons in human patients with callosal dysgenesis, being unable to cross the midline, would simply turn and project in a rostrocaudal direction, forming the Probst bundle. In contrast, later studies of Probst bundle fibers using tract-tracing agents such as horseradish peroxidase (Ozaki et al., 1987) or carbocyanine dyes (Ozaki and Wahlsten, 1993) in acallosal mice describe axons taking "whorled and convoluted paths" within the Probst bundles. Recently, diffusion tensor magnetic resonance imaging (DTMRI), which allows for the determination of both the location and the direction of large fiber tracts in the brain based on the anisotropic nature of water molecule diffusion within axon bundles, has been applied to the study of callosal dysgenesis in human patients (Lee et al., 2004; Tovar-Moll et al., 2007). Findings from these studies suggest that fibers run in a rostrocaudal manner within the Probst bundle, similar to the hypothesis put forth by Rakic and Yakovlev (1968).

Here, we use DTMRI to analyze the internal axonal makeup of the Probst bundles of Netrin1- and deleted in colorectal cancer 
(DCC)-deficient mice. Because diffusion tensor imaging (DTI) is ultimately an indirect means of visualizing fiber structures in space, it is very important to pair DTMRI studies with conventional histological or tract-tracing techniques to ensure that axonal tracts and their orientations identified by DTMRI scans are accurate. Here, we apply a combination of highresolution DTMRI analysis with immunohistochemical and carbocyanine dye tracttracing studies in mouse models of Probst bundle development to more precisely describe the microaxonal tract structure of Probst bundles. We use two mouse models that display complete agenesis of the corpus callosum and the formation of Probst bundles: mouse mutations in the chemotropic guidance molecule Netrin1 (Serafini et al., 1996) and its receptor DCC (Fazeli et al., 1997). Our results indicate that the pattern of fiber organization varies in different parts of the Probst bundle. In general, both DTMRI and tract-tracing analysis demonstrate that fibers within the dorsal region of the Probst bundle project rostrocaudally in an ordered manner, whereas fibers in the ventral region of the Probst bundle project in a far more disorganized manner.

\section{Materials and Methods}

Animals. Netrin1 and DCC mouse colonies were established from breeding pairs on a CD1 inbred background kindly provided by $\mathrm{M}$. Tessier-Lavigne (University of California at San Francisco, San Francisco, CA; and currently at Genentech Pharmaceuticals, San Francisco, CA). Before analysis, both strains were successively backcrossed onto a C57BL/6 background for $>10$ generations at University of Maryland, Baltimore, School of Medicine with the approval of the Institute Animal Care and Use Committee at The University of Maryland School of Medicine. Mice were imported to Australia and then bred and maintained at the animal facilities of The University of Queensland. Breeding and experiments were performed with the approval of the Animal Ethics Committee of The University of Queensland. Embryos from time-mated mice were collected at embryonic day 18 (E18), anesthetized on ice, and perfused transcardially with saline followed by $4 \%$ paraformaldehyde (PFA). Brains were stored in 4\% PFA until MRI scanning, and the same brains were used for immunohistochemical and tract-tracing analyses. A total of 43 E18 brains were analyzed in this study, including 8 Netrin1-/brains, 10 Netrin $1+/-$ brains, 11 DCC $-/-$ brains, 5 DCC $+/-$ brains, and 9 wild-type brains.

Immunohistochemistry. Perfusion-fixed brains were blocked in 3\% agar and cut into $45 \mu \mathrm{m}$ sections on a Vibratome (Leica, Nussloch, Germany). Immunostaining of floating sections using the axonalspecific mouse antibody growth cone-associated protein 43 (GAP43) (1:50,000; MAB 347; Chemicon, Temecula, CA) was processed as described previously (Shu et al., 2000). Mounted sections were coverslipped using DPX [distyrene, plasticizer (dibutylphthalate), and xylene] mounting medium and photographed using a high-resolution color camera mounted on a Zeiss (Thornwood, NY) Z1 upright microscope.

DTMRI. Imaging of E18 wild-type and DCC/Netrin 1 mutant brains was performed using an 11.7 tesla nuclear magnetic resonance spectrometer (Biospin; Bruker, Billerica, MA). Diffusion-weighted (DW) images were acquired with a three-dimensional DW fast spin echo sequence (Mori and van Zijl, 1998) [repetition time, $700 \mathrm{~ms}$; echo time, $27 \mathrm{~ms}$; echo train length, 4 ; diffusion time $(\Delta), 12 \mathrm{~ms}$; gradient duration $(\delta), 5$ $\mathrm{ms}$; diffusion gradient strength, 14 Gaussians/cm; imaging resolution, $0.09 \times 0.09 \times 0.09 \mathrm{~mm}$; signal averages of 4 ; two navigator echoes for eddy current correction]. Seven DW images with different $b$ values were acquired (one image with a $b$ value of $50 \mathrm{~s} / \mathrm{mm}^{2}$ and the rest with $b$ values of $1200 \mathrm{~s} / \mathrm{mm}^{2}$ ). Diffusion sensitizing gradients were applied along six different orientations: $[0.707,0.707,0],[0.707,0,0.707],[0,0.707$, 0.707], [ $-0.707,0.707,0],[0.707,0,-0.707],[0,-0.707,0.707]$. The total imaging time was $\sim 15 \mathrm{~h}$. The diffusion tensor was calculated using a Log-linear fitting method, with three pairs of eigenvalues and eigenvectors calculated for each pixel. The eigenvector associated with the largest eigenvalue was referred to as the primary eigenvector. For quantification of anisotropy, fractional anisotropy (FA) was used (Pierpaoli and Basser, 1996). Using the primary eigenvector and FA, color maps were calculated in which the color of each pixel is defined by the orientation of its primary eigenvector and the intensity of each pixel is proportional to its FA. Red was assigned to fiber orientation along the anteroposterior axis, green was assigned to the mediolateral axis, and blue was assigned to the dorsoventral axis. DTI-tractography was performed with DTI-Studio software using the technique called "fiber assignment by continuous tracking." To reconstruct the fiber tracts formed by callosal and hippocampal axons via DTI-tractography (Mori et al., 1999), two-dimensional regions of interest (ROIs) were placed within the Probst bundles and fimbria of DCC and Netrin1 mutant brains or the corpus callosum and hippocam- 


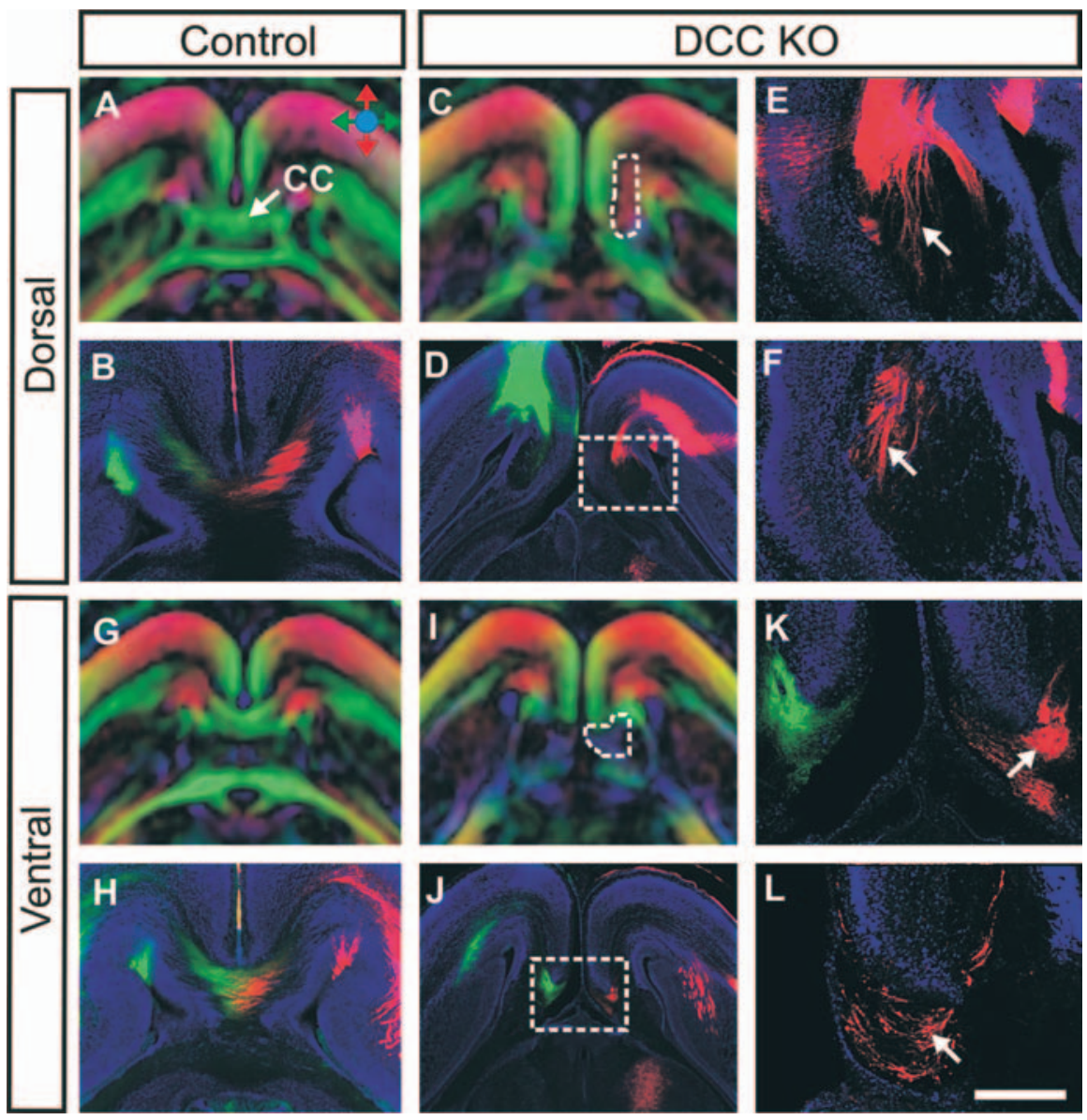

Figure 2. DTMRI and tract-tracing analysis of Probst bundles in DCC knock-outs. Using both DTMRI scanning and carbocyanine dye tract tracing, the fiber structure of Probst bundles was analyzed in E18 DCC knock-out (DCC KO) brains $(\boldsymbol{C}-\boldsymbol{F}, \boldsymbol{I}-\boldsymbol{L})$ and compared with wild-type controls $(\boldsymbol{A}, \boldsymbol{B}, \boldsymbol{G}, \boldsymbol{H})$. Colored arrows in $\boldsymbol{A}$ indicate fiber direction in DTMRI color maps, with the rostrocaudal direction in red, the mediolateral direction in green, and the dorsoventral direction in blue. $\boldsymbol{A}, \boldsymbol{B}, \boldsymbol{G}, \boldsymbol{H}$, In control embryos at E18, the corpus callosum (CC; in green) can be visualized in the horizontal view $(\boldsymbol{A}, \boldsymbol{G})$ and can be recapitulated via dye tract tracing $(\boldsymbol{B}, \boldsymbol{H}) . \boldsymbol{C}-\boldsymbol{F}, \boldsymbol{I}$, In DCC knock-out brains, Probst bundles $(\boldsymbol{C}, \boldsymbol{I}$, outlined by white dashed lines) form instead. Under DTMRI in the horizontal view, the dorsal part of the bundle is pseudo-colored red $(\boldsymbol{C})$, indicating fibers projecting in a rostrocaudal direction, and a large fiber bundle in the rostrocaudal axis can also be seen by Dil tract tracing $(\boldsymbol{E}, \boldsymbol{F}$, arrows; $\boldsymbol{E}$ is a higher-power view of the boxed area in $\boldsymbol{D})$. $\boldsymbol{I}-\boldsymbol{L}$, In contrast, the ventral region of the bundle is black by DTMRI, indicating fibers that do not project in any specific direction $(\boldsymbol{I})$, and under Dil tract tracing, Probst fibers indeed appear disorganized $(\boldsymbol{K}, \boldsymbol{L}$, arrows; $\boldsymbol{K}$ is a higher-power view of the boxed area in J). Scale bar: (in $\boldsymbol{L}) \boldsymbol{A}, \boldsymbol{C}, \boldsymbol{D}, \boldsymbol{G}, \boldsymbol{I}, \mathbf{J}, 1 \mathrm{~mm} ; \boldsymbol{B}, \boldsymbol{H}, 500 \mu \mathrm{m} ; \boldsymbol{E}, \boldsymbol{F}, \boldsymbol{K}, \boldsymbol{L}, 250 \mu \mathrm{m}$.

pal commissure of wild-type controls, and fibers were tracked from the ROIs using the following parameters: fiber tracking was restricted to voxels with a minimum FA of 0.35 , and the angle of deflection threshold between contiguous voxels was set at $50^{\circ}$.

Carbocyanine dye tracing. Dye injections were made using glass pipettes filled with $10 \%$ solutions of either $1,1^{\prime}$-dioctadecyl-3,3,3', $3^{\prime}$ tetramethylindocarbocyanine perchlorate (DiI) or 4-(4-(dihexadecyl amino)styryl)-N-methylpyridinium iodide (DiA) (Invitrogen, Eugene, OR) in dimethylformamide, attached to a pressure injector (Picospritzer; Parker Instrumentation, Carlstadt, NJ). To label callosal axons, one dye was injected into the frontal cortex of each hemisphere, and labeled brains were stored at $37^{\circ} \mathrm{C}$ in darkness for $>4$ weeks to allow for sufficient dye diffusion. Labeled brains were then blocked in 3\% agarose and serial sectioned at $100 \mu \mathrm{m}$ using a Vibratome (Leica). Sections were washed in PBS and counterstained for $30 \mathrm{~min}$ with DAPI (4'6-diamidino-2phenylindole dihydrochloride; 1:20,000 in PBS; Sigma, St. Louis, MO), before being mounted in a PVA-DABCO (polyvinyl alcohol-1,4diazabicyclo[2.2.2] octane) mounting medium (Sigma-Aldrich). Images were collected either on a fluorescent microscope (Zeiss Z1) fitted with an optical-sectioning system (Zeiss Apotome) or on a confocal microscope (Zeiss LSM510 Meta).

\section{Results}

Immunohistochemical analysis of Probst bundle structure

Netrin1 is a secreted guidance molecule and has been shown to play a key role in axon guidance during development (for review, see Barallobre et al., 2005). The loss of either Netrin 1 or its receptor DCC in mouse results in complete agenesis of all forebrain commissures including the corpus callosum, the hippocampal commissure, and the anterior commissure (Serafini et al., 1996; Fazeli et al., 1997). Misguided callosal fibers in the mutants form Probst bundles, and thus these mice serve as an excellent model system for the study of Probst bundle structure and development.

In the present study, we first analyzed the formation of Probst bundles in DCC- and Netrin1-deficient mice through immunohistochemistry with the axon-specific antibody GAP43. At E18, Probst bundles are present in both Netrin1 (Fig. $1 B, E$ ) and DCC (Fig. 1C,F) mutant brains, in contrast with the normally formed corpus callosum in control embryos (Fig. 1 $A, D$ ). In observing the trajectories of axons within the Probst bundle at higher magnification, and especially in the coronal plane, swirling fibers can be discerned in both Netrin 1 and DCC mutant mice (Fig. $1 B^{\prime}, C^{\prime}$, arrows), similar to the tortuous/convoluted pattern of fibers reported by Ozaki and colleagues (Ozaki et al., 1987; Ozaki and Wahlsten, 1993 ) in previous histological studies. However, it is difficult to discern the exact paths taken by single axons within the Probst bundle because the entire structure is immunostained.

\section{Analysis of Probst bundles using \\ DTMRI and dye tract tracing}

To more clearly define the internal axonal structure of the Probst bundles, we applied a combination of DTMRI scanning and carbocyanine dye tracttracing techniques to visualize axons. DTI is a powerful and noninvasive technique whereby the location and direction of major fiber tracts in the brain can be mapped after a three-dimensional scan of the brain and quantification of water molecule diffusion patterns on a voxel basis (for review, see Le Bihan, 2003). DTI measures the extent of water molecule diffusion along many directions. In axon fibers, the axonal membrane acts as barrier that reduces the extent of water diffusion crossing the fiber compared with along the fiber. DTI can detect and quantify this anistropic diffusion and give the orientation of maximal water diffusion (which is along the axon fibers), and a three-dimensional color map of the brain is generated, using a color-coding system to represent the orientation of axon projection. Because the contrasts generated by DTI are based on water diffusion, it cannot distinguish the retrograde or anterograde direction of axon bundles. In our study, high-resolution DTMRI scans of control embryos reveal a normally formed corpus callosum at E18 in a hor- 
izontal plane of view (Fig. $2 A, G$ ), and these results are verified by $\mathrm{DiI}$ and $\mathrm{DiA}$ tract-tracing experiments (Fig. $2 \mathrm{~B}, \mathrm{H}$ ).

Using DTMRI scanning, we were able to easily locate Probst bundles in both DCC (Fig. 2C,I) and Netrin1 (Fig. 3B-D) mutant mice at E18. Our results suggest that in the dorsal part of the Probst bundle (Figs. $2 C, 3 B, C$ ), axons project rostrocaudally within the bundle (as indicated by the Probst bundle being pseudo-colored red in the scan), similar to the DTMRI findings of Probst bundle structure in human acallosal patients (Lee et al., 2004). Using tract tracing, DiI-labeled fibers within the dorsal region of the bundle indeed form bundles that project in a rostrocaudal direction in both DCC-deficient (Fig. 2E,F, arrows) and Netrin1-deficient (Fig. $3 F^{\prime}, G^{\prime}$, arrows) mice, confirming our DTMRI results. In contrast, DTMRI sections showing more ventral parts of the Probst bundle reveal vastly different diffusion patterns compared with the dorsal regions. The ventral part of the bundle is dark blue or black (Figs. $2 I$, outlined area, 3D), and black on a DTMRI color map indicates either non-axonal structures (such as within a ventricle) or a region where fibers project in multiple directions, thereby nullifying each other's signal (Le Bihan, 2003). After dye tract tracing, we found that DiIlabeled fibers are present in this part of the bundle, but their trajectories appear random and disorganized (Figs. $2 \mathrm{~K}, \mathrm{~L}, 3 \mathrm{H}^{\prime}$, arrows). This pattern of axon projections fits with the "convoluted" description given to fibers in previous studies of Probst bundle structures (Ozaki et al., 1987; Ozaki and Wahlsten, 1993).

\section{Probst fiber reconstruction using DTI-tractography}

In addition to merely visualizing the locations of fiber bundles in the brain, DTMRI can also be used to reconstruct axon tracts originating from specific ROIs. This is termed "DTI-tractography" and serves as a form of noninvasive tract tracing (Mori et al., 1999). DTItractography has proven to be particularly useful for studying axonal pathways in human subjects where histological tract tracing is not possible. A recent study by Tovar-Moll et al. (2007) used this technique to analyze the structure of Probst bundles in a cohort of human patients with callosal dysgenesis and found that the Probst bundle is topographically organized and projects in a rostrocaudal direction. Here, we take a similar approach in using DTItractography to complement our DTMRI and dye-tract tracing studies of Probst bundle structure.

First, we applied DTI-tractography to visualize the corpus callosum (CC) and hippocampal commissure (HC) projections in wild-type controls. After placing two ROIs in a midsagittal section (Fig. 4A, CC in white, HC in yellow), both commissures were reconstructed via DTI-tractography. Callosal fibers can be traced retrogradely from the callosal bundle, projecting dorsally into the cortex along the entire length of the corpus callosum (Fig. 4D). Hippocampal commissure fibers can be traced back to the dentate gyrus (Fig. 4G, arrow). In the DCC- and Netrin1-deficient brains, ROIs were placed in horizontal or coronal sections rather than a mid-sagittal
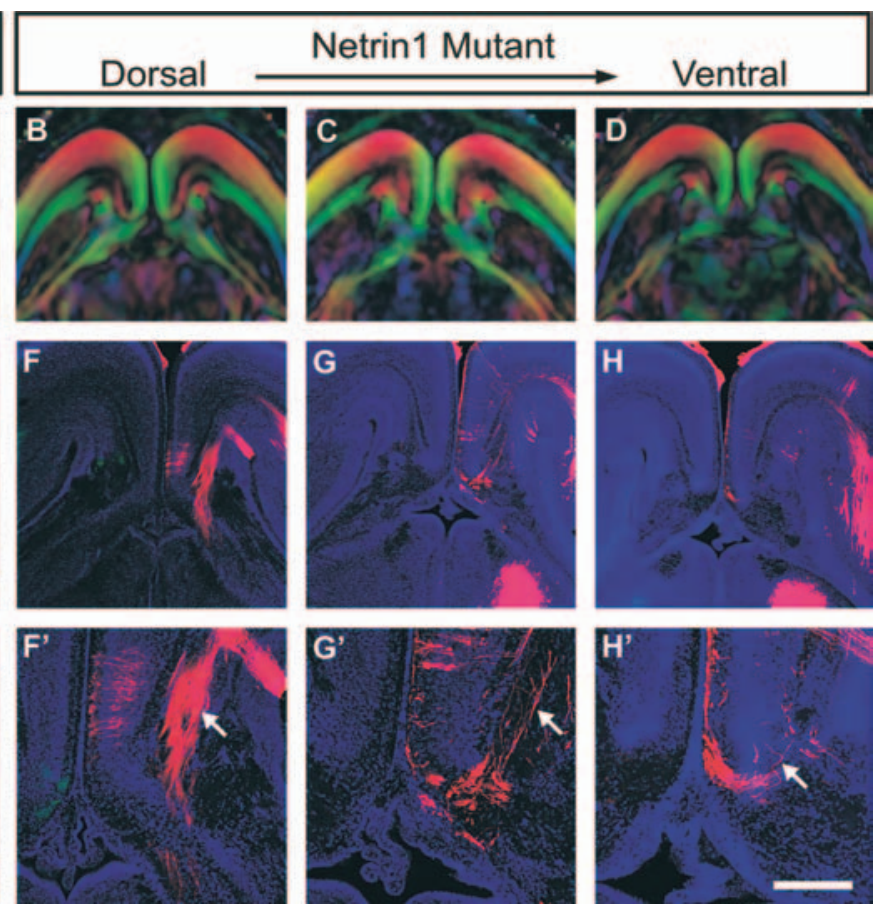

Figure 3. DTMRI and tract-tracing analysis of Probst bundles in Netrin1 mutants. Using both DTMRI scanning and carbocyanine dye tract tracing, the fiber structure of Probst bundles were analyzed in E18 Netrin1 mutant brains $(\boldsymbol{B}-\boldsymbol{D}, \boldsymbol{F}-\boldsymbol{H}$; magnified in $\left.-\boldsymbol{H}^{\prime}\right)$ and compared with wild-type controls $\left(\boldsymbol{A}, \boldsymbol{E}, \boldsymbol{E}^{\prime}\right)$. Similar to the DCC knock-outs, Probst bundles are pseudo-colored red in racing $\left(\boldsymbol{F}^{\prime}, \boldsymbol{G}^{\prime}\right.$, arrow). In contrast, Dil-labeled fibers in the ventral region of the Probst bundle project in a disorganized manner $\left(\boldsymbol{H}^{\prime}\right.$, arrow). Scale bar: (in $\left.\boldsymbol{H}^{\prime}\right) \boldsymbol{A}-\boldsymbol{D}, 1 \mathrm{~mm} ; \boldsymbol{E}-\boldsymbol{H}, 600 \mu \mathrm{m} ; \boldsymbol{E}^{\prime}-\boldsymbol{H}^{\prime}, 300 \mu \mathrm{m}$.

section because no commissural fibers cross the midline in these mice. In horizontal sections at the level of the Probst bundle, ROIs were placed bilaterally within the Probst bundle (Fig. $4 B, C$, white) to trace callosal axons from the cortex and within the fimbria to trace hippocampal axons. In both mutant strains, hippocampal fibers (Fig. $4 E, F, I$, yellow) emerge from the dentate gyrus normally but fail to cross the midline. DTI-tractography was, however, able to trace some of the ipsilaterally projecting hippocampal fibers as they enter the fornix (Fig. $4 F$, arrow). Cortical fibers (in white) also fail to cross the midline, but instead of merely pausing at the midline like the hippocampal fibers, they form the large Probst bundles, which extend rostrocaudally near the midline but do not intermingle with hippocampal or fornix axons (best appreciated in the sagittal view in Fig. $4 H$ ).

One of the advantages of DTI-tractography is the ability to reconstruct all of the axon bundles that form connections through the target ROI. We found that axons entering the Probst bundles originate not only from the frontal lobe (Fig. $4 E$, white arrow), where we previously performed carbocyanine dye tract tracing, but also from medial (Fig. $4 E$, blue arrow) and lateral (Fig. $4 E$, red arrow) aspects of parietal cortex as well, the most caudal area where callosal axons have crossed the midline by E18 (the oldest age these mice survive to). Finally, our DTI-tractography results reconfirm our previous DTI and dye tract-tracing findings of fibers projecting rostrocaudally within the dorsal Probst bundle, whether fibers are viewed in the sagittal view (Fig. $4 H$ ) or emerging out of the visual plane in the coronal view (Fig. 4I).

\section{Discussion and Conclusions}

Agenesis of the corpus callosum and other forms of callosal dysgenesis occur in 1 in 4000 live births, and the formation of Probst 


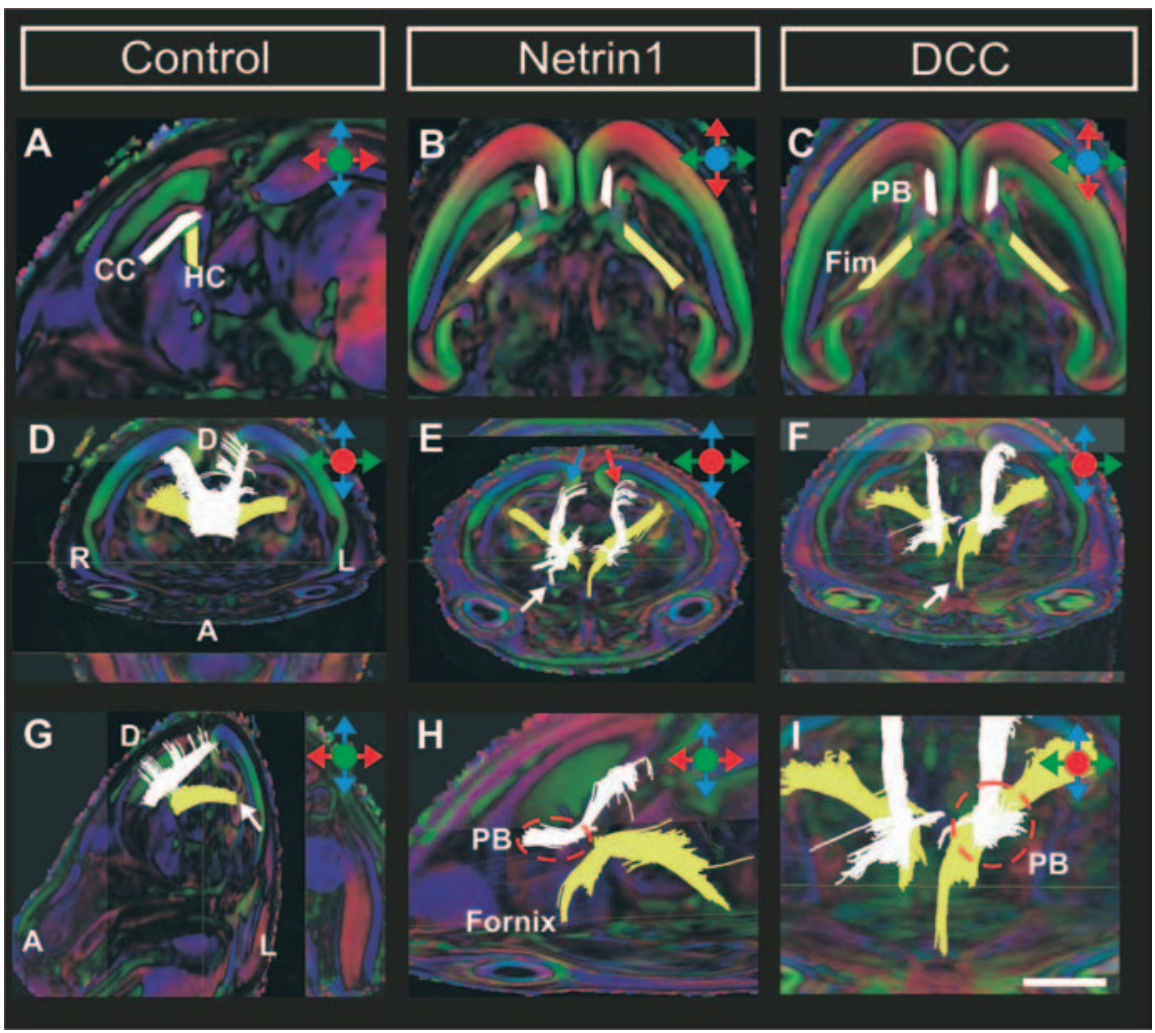

Figure 4. DTI-tractography in DCC and Netrin1 mutant brains. Trajectories of fibers entering and traveling within the corpus callosum or Probst bundle (in white) and the hippocampal commissure (in yellow). $\boldsymbol{A}-\boldsymbol{C}$, ROls used for DTI-tractography. $\boldsymbol{D}, \mathbf{G}$, In wild-type controls, cortical fibers cross the midline to form the corpus callosum $(\boldsymbol{D})$, whereas fibers emerging from the dentate gyrus ( $G$, arrow) cross to form the hippocampal commissure. $\boldsymbol{E}, \boldsymbol{F}$, In DCC and Netrin 1 mutant brains, hippocampal fibers from the fimbria either terminate at the midline or project ipsilaterally into the fornix $(\boldsymbol{F}$, arrow), whereas cortical fibers form bilateral Probst bundles $(\boldsymbol{E}, \boldsymbol{F})$. The Probst bundle comprises of axons originating from throughout the cortex $(\boldsymbol{E}$, arrows). $\boldsymbol{H}, \boldsymbol{I}$, Within the $\mathrm{PB}$, fibers project in a rostrocaudal direction (white tract) and remain separate from the hippocampal fibers (yellow tract). $I$ is a higher-power view of $F$. CC, Corpus callosum; $\mathrm{HC}$, hippocampal commissure; $\mathrm{PB}$, Probst bundle; Fim, fimbria. Orientation of the brain is as follows: $R$, right; L, left; $A$, anterior; $D$, dorsal. Scale bar: (in $I) A-C, 1 \mathrm{~mm} ; \boldsymbol{D}-\mathbf{G}, 1.5 \mathrm{~mm} ; \boldsymbol{H}, \boldsymbol{I}, 750 \mu \mathrm{m}$.

bundles is the most common structural anomaly associated with callosal dysgenesis. The advent of DTMRI technology has offered the first glimpses of the internal structure of Probst bundles in humans (Lee et al., 2004; Tovar-Moll et al., 2007). In the present study, by combining DTMRI analysis with immunohistochemistry and a carbocyanine tract-tracing experiment in animal models of Probst bundle formation, we have obtained the clearest view yet of how axons traverse within the bundle itself. The combined use of high-resolution DTMRI scanning and focal carbocyanine dye injections (so that individual fibers, rather than the entire Probst bundle, are labeled) have revealed that axons behave differently in different parts of the corpus callosum. The dorsal (and more rostral) region of the Probst bundle comprises organized axon bundles projecting rostrocaudally, whereas the ventral (and more caudal) region of the Probst bundle, where cortical fibers presumably terminate after failing to cross the midline, exhibits a far more disorganized structure. This suggests that some axon guidance activity may be retained in the dorsal Probst bundle, allowing cortical fibers to project in an ordered array even if they ultimately fail to cross the midline. Finally, although our dye tract tracing is limited to fibers from the frontal lobe, the use of DTI-tractography has shown that the Probst bundle is comprised of fibers originating from throughout the cortex. To- gether, these results give greater insight into how misguided callosal axons coalesce into the large aberrant fiber structures known as Probst bundles.

\section{References}

Barallobre MJ, Pascual M, Del Rio JA, Soriano E (2005) The Netrin family of guidance factors: emphasis on Netrin-1 signalling. Brain Res Brain Res Rev 49:22-47.

Fazeli A, Dickinson SL, Hermiston ML, Tighe RV, Steen RG, Small CG, Stoeckli ET, Keino-Masu K, Masu M, Rayburn H, Simons J, Bronson RT, Gordon JI, Tessier-Lavigne M, Weinberg RA (1997) Phenotype of mice lacking functional deleted in colorectal cancer (DCC) gene. Nature 386:796-804.

Le Bihan D (2003) Looking into the functional architecture of the brain with diffusion MRI. Nat Rev Neurosci 4:469-480.

Lee SK, Mori S, Kim DJ, Kim SY, Kim DI (2004) Diffusion tensor MR imaging visualizes the altered hemispheric fiber connection in callosal dysgenesis. Am J Neuroradiol 25:25-28.

Mori S, van Zijl PC (1998) A motion correction scheme by twin-echo navigation for diffusionweighted magnetic resonance imaging with multiple RF echo acquisition. Magn Reson Med 40:511-516.

Mori S, Crain BJ, Chacko VP, van Zijl PC (1999) Three-dimensional tracking of axonal projections in the brain by magnetic resonance imaging. Ann Neurol 45:265-269.

Onufrowicz, W (1888) Das balkenlose microcephalengehirn Hofmann. Arch Psychiatr Nervenkr 18: 305-328.

Ozaki HS, Wahlsten D (1993) Cortical axon trajectories and growth cone morphologies in $\mathrm{fe}$ tuses of acallosal mouse strains. J Comp Neurol 336:197-206.

Ozaki HS, Murakami TH, Toyoshima T, Shimada M (1987) The fibers which leave the Probst longitudinal bundle seen in the brain of an acallosal mouse: a study with the horseradish peroxidase technique. Brain Res 493:66-73.

Paul LK, Brown WS, Adolphs R, Tyszka JM, Richards LJ, Mukherjee P, Sherr EH (2007) Agenesis of the corpus callosum: genetic, developmental and functional aspects of connectivity. Nat Rev Neurosci 8:287-299.

Pierpaoli C, Basser PJ (1996) Toward a quantitative assessment of diffusion anisotropy. Magn Reson Med 36:893-906.

Probst M (1901) Ueber den Bau des balkenlosen grosshirns, sowie uber mikrogirie und heterotopie der grauen substanz. Arch Psychiatr Nervenkr 34:709-786.

Rakic P, Yakovlev PI (1968) Development of the corpus callosum and cavum septi in man. J Comp Neurol 132:45-72.

Richards LJ, Plachez C, Ren T (2004) Mechanisms regulating the development of the corpus callosum and its agenesis in mouse and human. Clin Genet 66:276-289.

Serafini T, Colamarino SA, Leonardo ED, Wang H, Beddington R, Skarnes WC, Tessier-Lavigne M (1996) Netrin-1 is required for commissural axon guidance in the developing vertebrate nervous system. Cell 87:1001-1014.

Shu T, Valentino KM, Seaman C, Cooper HM, Richards LJ (2000) Expression of the Netrin-1 receptor, deleted in colorectal cancer (DCC), is largely confined to projecting neurons in the developing forebrain. J Comp Neurol 416:201-212.

Tovar-Moll F, Moll J, Oliveira-Souza R, Bramati I, Andreioulo PA, Lent R (2007) Neuroplasticity in human callosal dysgenesis: a diffusion tensor imaging study. Cereb Cortex 17:531-541. 